\title{
Posterior Scleritis Simulating Choroidal Melanoma: A Case Report
}

\author{
(1) Simanta Khadka, (1) Raghunandan Byanju, (1) Sangita Pradhan \\ Department of Vitreo-Retina, Bharatpur Eye Hospital, Chitwan, Nepal
}

\begin{abstract}
Nodular posterior scleritis (NPS) is rare; however, it is critical to differentiate it from similar intraocular pathologies to prevent inappropriate and potentially irreversible treatment. Multimodal imaging is beneficial in the diagnosis of NPS. This report describes the diagnosis and management of a case of NPS with corticosteroid therapy that led to complete resolution.
\end{abstract}

Keywords: Choroidal melanoma, corticosteroids, nodular posterior scleritis, simulating lesion

\section{Introduction}

Posterior scleritis is an uncommon and under-recognized form of inflammatory disease of the sclera (I). The relative unfamiliarity of the general ophthalmologist and inconsistent manifestations often lead to misdiagnosis of this condition $(2,3)$. The underlying mechanism of posterior scleritis also remains elusive and the majority of the research has focused on characterizing its clinical features and optimizing the diagnosis, treatment, and patient outcome (4). Posterior scleritis accounts for some $10 \%$ of all cases of scleritis and is associated with systemic diseases in as many as one-third of cases (5). There are 2 variants: diffuse posterior scleritis and nodular posterior scleritis (NPS) (I). The diffuse form leads to generalized inflammation of the sclera and increased thickness of the eyewall, while the nodular subtype produces a localized scleral nodule. The latter usually results in an amelanotic subretinal mass lesion with overlying subretinal fluid and chorioretinal folds. Since NPS is rare, it may be confused with or even misdiagnosed as an intraocular tumor, such as granuloma, hemangioma, or even choroidal melanoma or posterior uveitis (6). It is important to differentiate NPS from choroidal melanoma because clinical misdiagnosis can lead to misdirected treatment, including enucleation (7).

Despite growing experience and advancement in diagnostic modalities, NPS continues to be a diagnostic enigma, as it can often simulate choroidal melanoma clinically (8). Familiarity and a high index of suspicion are necessary to detect this potentially sight-threatening disease early in its course in order to administer effective therapy to limit visual loss and prevent misguided therapy.

This case of NPS closely resembled amelanotic choroidal melanoma. The diagnosis was established based on clinical findings and multi-modal imaging techniques. The condition resolved completely following corticotherapy.

\section{Case Report}

A 52-year-old male presented with the complaints of diminution of vision in the left eye (LE) accompanied by mild

How to cite this article: Khadka S, Byanju R, Pradhan S. Posterior Scleritis Simulating Choroidal Melanoma: A Case Report. Beyoglu Eye J 202 I; 6(2): I33-I39.

Address for correspondence: Simanta Khadka, MD. Department of Vitreo-Retina, Bharatpur Eye Hospital, Chitwan, Nepal

Phone: 9841572286 E-mail: simantakhadka@gmail.com

Submitted Date: January 24, 2021 Accepted Date: March 22, 2021 Available Online Date: June 08, 2021

${ }^{\circ}$ Copyright 2021 by Beyoglu Eye Training and Research Hospital - Available online at www.beyoglueye.com OPEN ACCESS This work is licensed under a Creative Commons Attribution-NonCommercial 4.0 International License. 
periorbital pain and a headache of I week duration (September 2019). His past medical and ocular history was unremarkable. On examination, his best corrected visual acuity (BCVA) was 6/6 in the right eye (RE) and 6/I2 in the LE. Extraocular motility was full in all cardinal gazes, and no proptosis was present. The pupils were normal with no relative afferent pupillary defect. The results of a slit-lamp examination of both eyes were within normal limits and there were no signs of anterior or vitreous inflammatory cells. The intraocular pressure measurement was also within normal limits. The RE fundus was unremarkable (Fig. IA). A dilated fundus examination of the LE revealed a large, central, dome-shaped, nonpigmented subretinal lesion temporal to the disc measuring approximately 6 disc diameters covering an area within the temporal arcades with an accumulation of subretinal fluid (Fig. IB, C, D). There was no evidence of retinal hemorrhage, pigmentary changes, or disc edema.

An Amsler's grid test of the LE revealed a central scotoma with distortion of lines in that region. Diagnostic B-scan ultrasonography (US) revealed a homogenous mass lesion temporal to the optic nerve head with medium surface reflectivity and moderate to low internal reflectivity. The lesion measured $8.6 \mathrm{~mm}$ in the largest basal diameter and 4.25 $\mathrm{mm}$ in elevation. Shallow exudative detachment and the absence of choroidal excavation or thickened adjacent sclera were observed (Fig. 2). However, no definitive $\mathrm{T}$ sign was noted. Optical coherence tomography (OCT) examination revealed multifocal exudative retinal detachment with increased choroidal thickening (Fig. 3). Both fundus fluorescein angiography (FFA) and indocyanine green angiography (ICGA) revealed blocked hypofluorescence of the choroidal mass with multiple leakage sites within and surrounding the mass. There was no evidence of double circulation. FFA showed early pinpoint areas of hyperfluorescence, along with late pooling of dye under the detached retina. ICGA demonstrated diffuse hypofluorescence corresponding to the area of exudative detachment in the early phase with multiple pinpoint hyperfluorescence in the late phase (Fig. 4).

Magnetic resonance imaging (MRI) of the orbit (plain and contrast) showed a focal, elevated, enhancing lesion in the temporal part of the left eyeball bulging into the vitreous cavity. The lesion demonstrated diffuse hyperintensity in TI-weighted scans (Fig. 5 A, B) and hypointesity in T2 images (Fig. $5 \mathrm{C}$ ). Post contrast, there was diffuse enhancement of the lesion and focal enhancement of the overlying periocular soft tissue (Fig. 5 D).

The initial workup comprised a detailed uveitis examination, which yielded negative results on a purified protein derivative intradermal skin test; autoimmune serological screening (rheumatoid factor, antinuclear antibodies, and antineutrophil cytoplasmic antibodies); hepatitis serology; and HIV and syphilis tests. Radiological tests performed in-
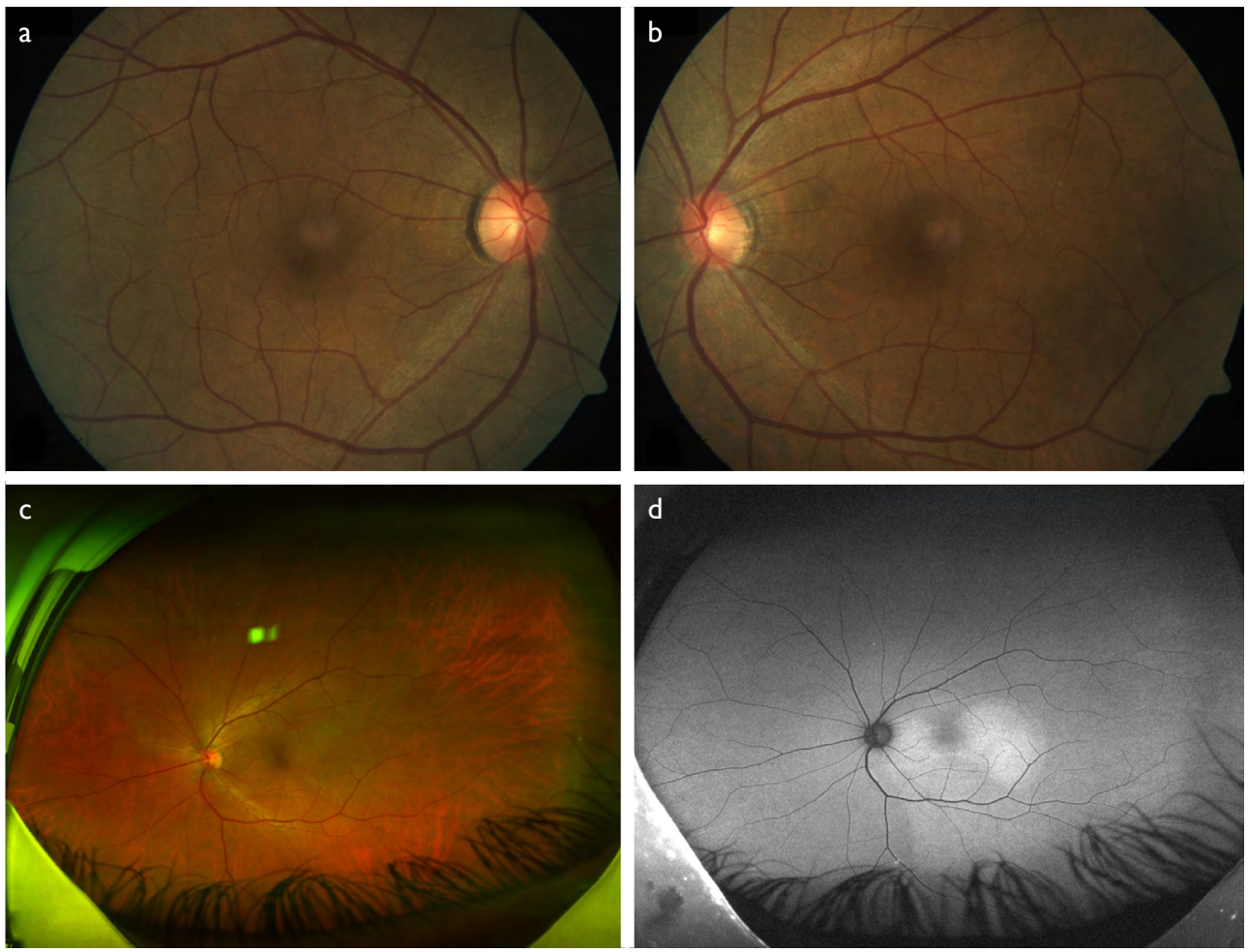

Figure I. Fundus photography. (a) Right eye within normal limits, (b) left eye with visible mass located inside the boundary of the temporal arcade, (c) wide field photography of the left eye, (d) fundus autofluorescence of the left eye revealing the mass lesion with autofluorescent properties. 

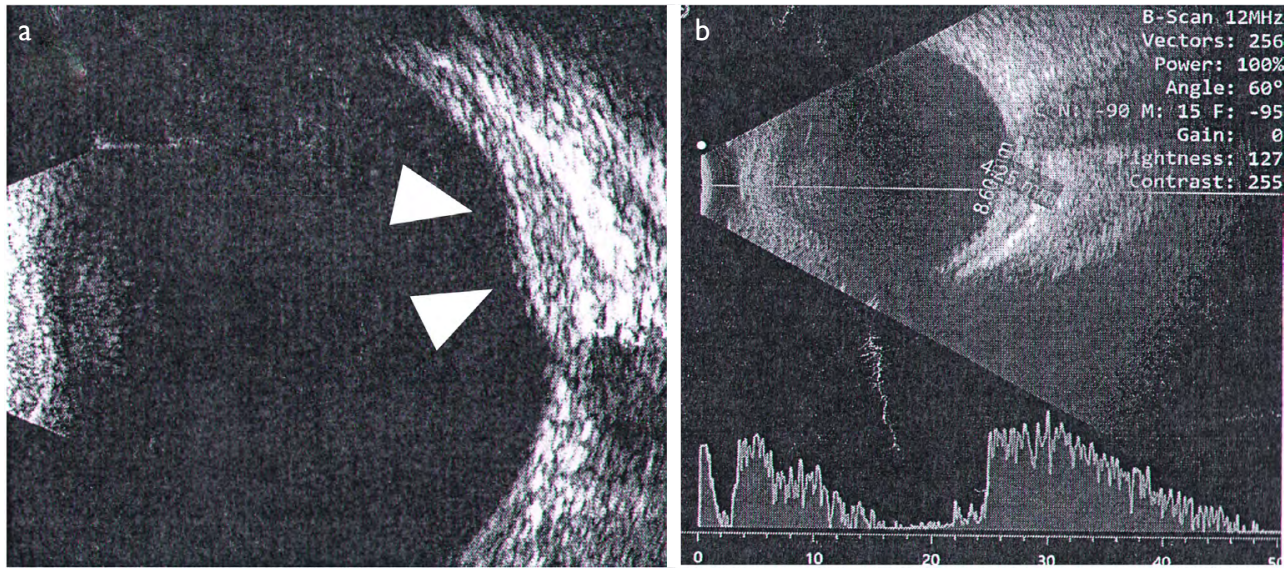

Figure 2. B scan ultrasonographic image of the mass lesion. (a) The lesion can be seen temporal to the disc (white arrowheads), (b) largest dimension of the mass lesion.

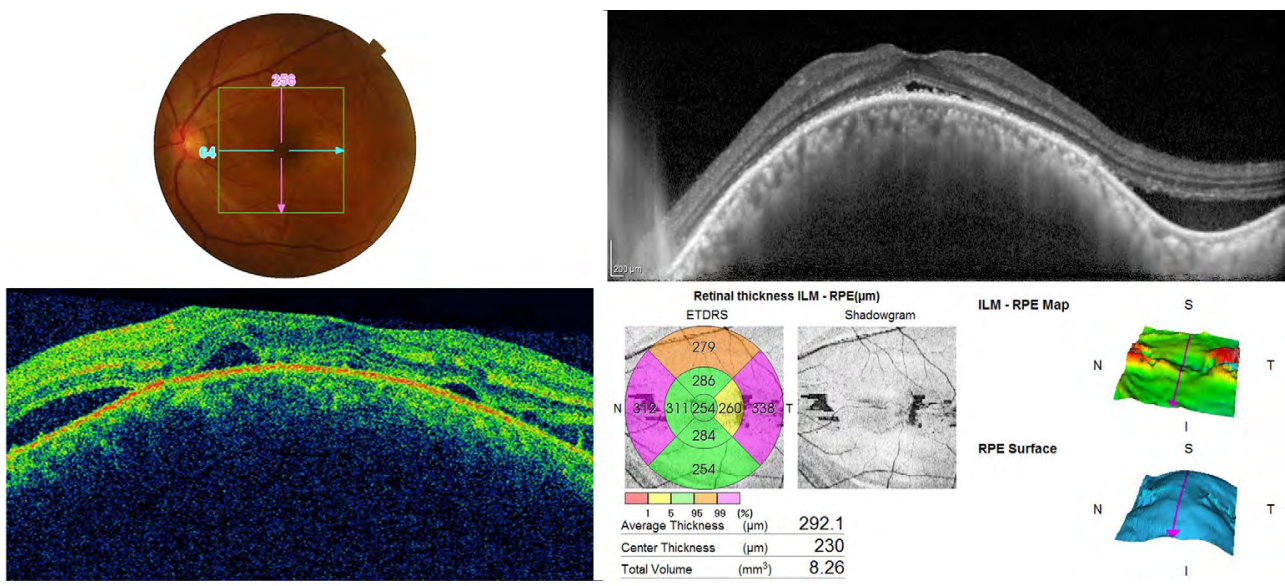

Figure 3. Optical coherence tomography of the left eye illustrating the dome-shaped solitary nodular lesion and multifocal exudative detachment of the retina.

cluded a chest X-ray, a chest computed tomography (CT) scan, and MRI of the brain, which produced unremarkable results. The absence of hilar lymphadenopathy and focal nodular or infiltrative lung parenchymal densities on chest CT imaging, and a normal serum angiotensin-converting enzyme level ruled out the possibility of ocular sarcoidosis. The optic disc and extraocular muscles appeared normal on an MRI of the left orbit, which excluded choroidal lymphoma. Moreover, the clinical history, examination, and diagnostic imaging confirmed the exclusion of primary central nervous system lymphoma, which can present with ocular vitritis or retinal pigment epithelium infiltrate; and secondary intraocular lymphoma, which can present as a part of systemic non-Hodgkin's B cell lymphoma with systemic manifestations and choroidal infiltration, which were not present. Similarly, a metastatic workup yielded unremarkable results.

The nonpigmented appearance of the lesion and normal intrinsic choroidal vasculature along with B-scan US and MRI findings were suggestive of NPS. Pulse steroid therapy was initiated with an injection of I gram intravenous methyl- prednisolone for 3 days followed by oral prednisolone 60 $\mathrm{mg}$ once daily tapered over 6 weeks. The patient noticed a decrease in the severity of the periorbital pain following the intravenous steroid injection and an improvement in vision at the conclusion of oral steroid therapy. During the course of treatment, the patient had no adverse drug-related side effects and the recovery was uneventful. Follow up was conducted for a year following the diagnosis, and at the end of the year (October 2020), the choroidal lesion had completely regressed (Fig. 6). There was no recurrence and the patient regained a BCVA of $6 / 6$ in the LE.

\section{Discussion}

Posterior scleritis is defined anatomically as inflammation of the sclera posterior to the ora serrata (I). The nodular variant of posterior scleritis is a distinct sclerochoroidal thickening, which can masquerade as an intraocular tumor. Though benign, this rare entity poses a considerable diagnostic dilemma (10). This inflammatory ocular disease is often associated with systemic autoimmune conditions, like rheumatoid 

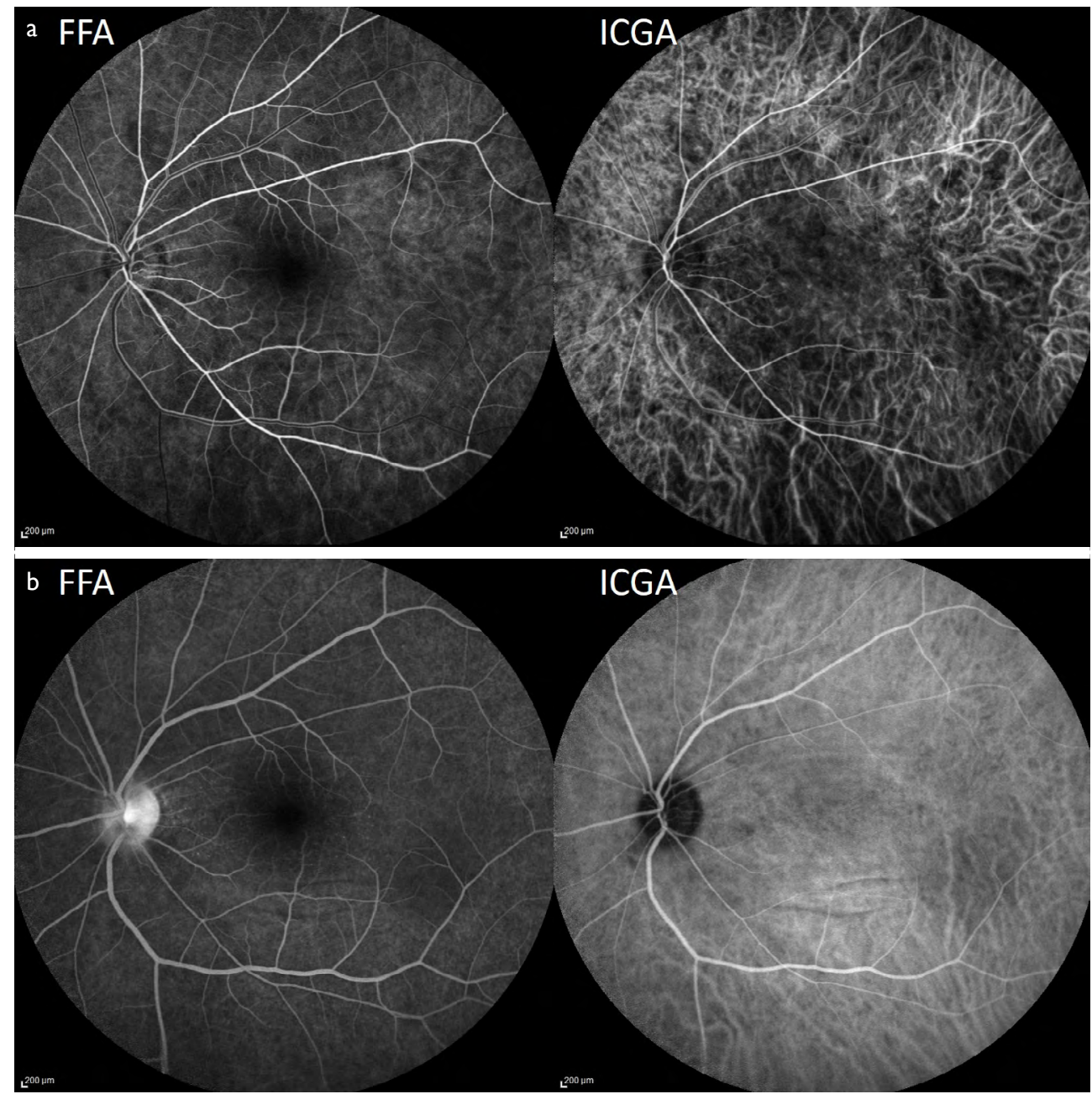

Figure 4. Fundus fluorescein angiography (FFA) and indocyanine green angiography (ICGA). (a) Early-phase images reveal blocked hypofluorescence of the choroidal mass with multiple leakage sites within and surrounding the mass. (b) Late-phase images show diffuse hypofluorescence with multiple pinpoint leakage sites seen with FFA and pooling of dye with ICGA, suggestive of exudative detachment.
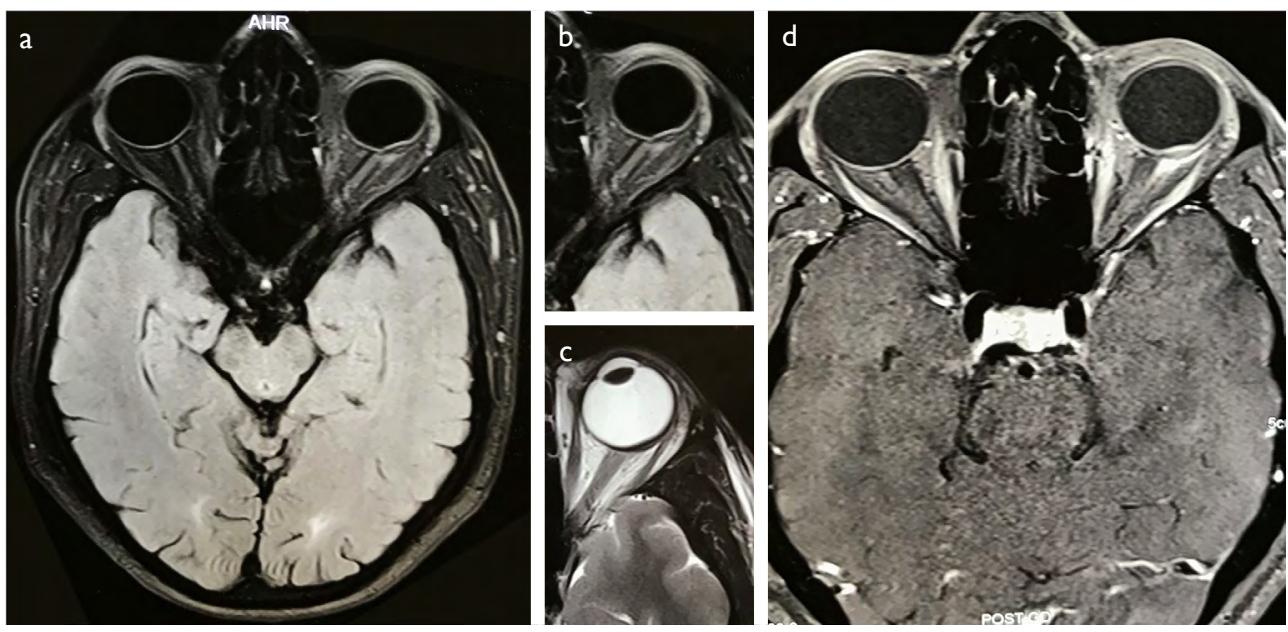

Figure 5. Magnetic resonance imaging of the orbit. (a, b) TI-weighted hyperintensity of the lesion, (c) T2-weighted image revealing hypointense mass lesion, (d) post gadolinium contrast image showing diffuse enhancement of the lesion as well as enhancement of surrounding periocular tissue posterior to the globe. 

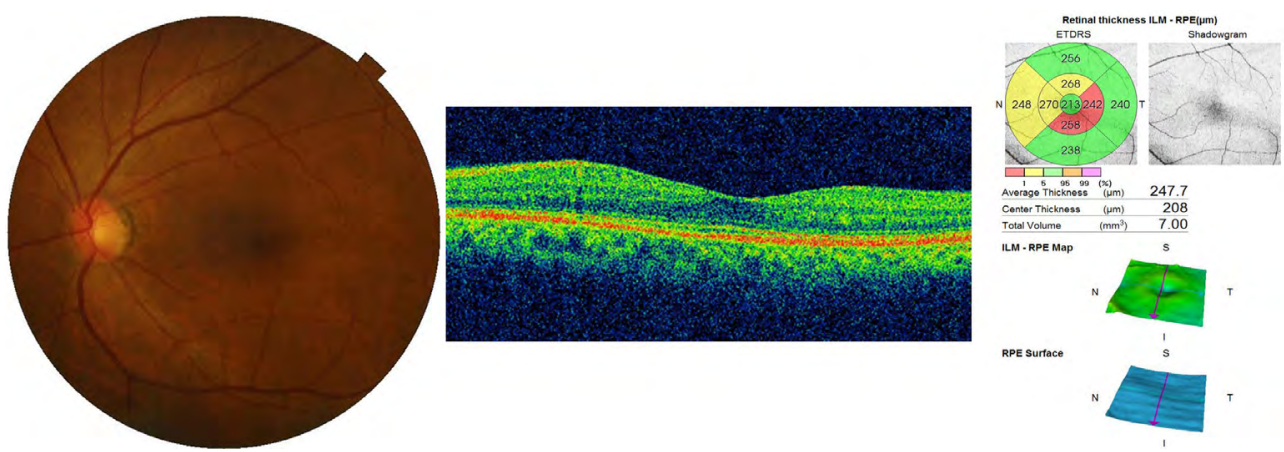

Figure 6. Fundus photograph and corresponding optical coherence tomography of the left eye after I year. The lesion has completely regressed and near-normal restoration of the foveal anatomy was achieved.

arthritis, relapsing polychondritis, psoriatic arthritis, and systemic lupus erythematosus (II). Rheumatoid arthritis is a frequent comorbidity $(5,9)$. However, the majority of cases may not exhibit any associated systemic abnormalities (6).

A review of the literature indicates that the mean age of an NPS patient at presentation is $\mathbf{5 2}$ years. The condition has been described as unilateral, and there was a female preponderance $(6,10)$. The commonly reported presenting complaints of patients with NPS are periocular pain and headache. The patient's vision may or may not be reduced, and posterior scleritis is usually accompanied by anterior scleritis (5). The age and presenting complaints of the patient in our case were consistent with the available literature; however, there were no signs of involvement of the anterior sclera.

B-scan US plays a pivotal role in differentiating a posterior tumor from other similar conditions. Malignant choroidal melanomas will demonstrate mixed echogenicity with mainly medium and low amplitude echoes, a collar-stud shape, or both features (12). However, a solitary lesion is present in NPS. The presence of edema in the Tenon capsule ( $T$ sign), (7) and choroidal effusion (5) signify inflammation, which may occur in NPS. However, these signs of inflammation were not evident in our case.

The ability of OCT to detect macular edema and determine the amount of subretinal fluid in posterior scleritis is somewhat limited, due to the location and size of the lesion (I0). MRI serves as an adjunct diagnostic modality to differentiate the inflammatory process from a malignant lesion (13). The MRI findings in our case particularly suggested choroidal melanoma based on the nodular mass, which was hyperintense in $\mathrm{Tl}$-weighted series and hypointense in T2-weighted series. These findings were similar to those of Saatci et al. (14) The hypointensity on T2 images and hyperintensity on $\mathrm{TI}$-weighted scans could be attributed to the paramagnetic nature of the lesion due to the accumulation of inflammatory cells, subacute blood products, or melanin. Similarly, the diffuse enhancement of the lesion following contrast is consistent with a thickened sclerouveal coat. Retinal detachment can occur as a result of exudation from the subretinal mass of nodular scleritis $(3,7)$. A linear contrast enhancement within the globe may indicate a detached retina or a displaced retina due to thickened sclera and choroidal layers (14).

A point that countered a diagnosis of intraocular malignancy in our case was the presence of periocular pain. Melanoma is generally a painless entity, but retinal symptoms of photopsia or blurred vision may occur (10). Melanoma tumor necrosis may lead to pain due to associated episcleritis and scleritis $(15,16)$. Similarly, melanoma is pigmented in approximately $85 \%$ cases and lipofuscin deposits are a sign of tumor growth (17). The amelanotic variant tends to have metastatic deposits and a larger amount of exudative fluid (18). The metastatic workup was negative in our patient. In addition, the ocular echographic findings further contradicted intraocular malignancy; a solitary nodule with internal reflectivity was evident in our case. The echography of choroidal melanoma reveals either a dome-shaped or a collar stud-shaped configuration when it ruptures Bruch's membrane (10). A fluorescein angiographic pattern of choroidal melanoma in which the tumor blood vessels begin to fluoresce early and leak progressively throughout the study or a characteristic double circulation pattern when the tumor has broken through Bruch's membrane (19) was not present in our study. When a diagnostic dilemma is present, a therapeutic trial of systemic steroids can help resolve the issue. Posterior scleritis usually responds dramatically to steroids, while neoplastic lesions are unlikely to respond.

The diagnosis of NPS is essentially clinical and B-scan US is considered an important diagnostic modality $(4,5,10,20)$. Additional information to exclude other simulating conditions is provided by adjunctive investigative techniques, such as FFA (6) and MRI $(13,14)$. Other differentials that should be considered for an amelanotic mass lesion include choroidal melanoma, choroidal metastasis, and choroidal heman- 
gioma (3). A few reports have described a similar kind of lesion associated with systemic lupus erythematosus $(\mathrm{I})$ and giant cell arteritis (2I). A scleral biopsy in a similar scenario revealed a thickened sclera infiltrated by chronic inflammatory cells (lymphocytes and eosinophils). A granulomatous reaction, an increase in fibroblasts, and areas of necrosis may also be observed $(7,13)$.

The underlying etiology, whether autoimmune, infectious, or neoplastic, commonly guides the treatment of posterior scleritis. Treatment options vary depending on the degree of inflammation, ranging from low potency non-steroidal anti-inflammatory drugs to more potent steroidal drugs, in either systemic or locally injectable forms, or other strong immunosuppressive agents (22). Systemic corticotherapy is the mainstay of treatment $(3,5)$. We managed our case with an injectable pulse steroid combined with tapered oral steroid therapy. Mycophenolate mofetil has also been used as a steroid-sparing agent (6). The prognosis of NPS is excellent and to the best of our knowledge, no recurrences have been reported in the literature (6). In some cases, partial regression has been achieved rather than complete resolution (10). The longest reported follow-up of a patient with giant NPS was a period of 12 years, and observation revealed a stable lesion with no effect on vision.

Clinical misinterpretation of NPS as a malignant melanoma may lead to erroneous therapy with potentially significant outcomes, including enucleation. When a choroidal mass presents with atypical features of melanoma, any evidence of inflammation may suggest inflammatory origin rather than a malignant cause. A trial of anti-inflammatory medication may serve as a therapeutic test and help spare patients from invasive and unnecessary interventions. NPS is a rare unilateral disease and may mimic an intraocular tumor clinically. It can cause a subretinal mass and exudative retinal detachment. Multimodal imaging, including US, OCT, FFA, ICGA, and MRI of the orbit should be utilized to confirm the diagnosis. The disease must be diagnosed correctly to avoid unnecessary workups and aggressive management. Most cases have an excellent prognosis with no recurrence.

\section{Disclosures}

Informed consent: Written informed consent was obtained from the patient for the publication of the case report and the accompanying images.

Peer-review: Externally peer-reviewed.

Conflict of Interest: None declared.

Authorship Contributions: Involved in design and conduct of the study (SK); preparation and review of the study (SK, RNB, SP); data collection (SK).

\section{References}

I. Sin PY, Liu DT, Young AL. Nodular posterior scleritis mimicking choroidal tumor in a patient with systemic lupus erythematous: a case report and literature review. Asia Pac J Ophthalmol (Phila) 2016;5:324-9. [CrossRef]

2. Biswas J, Mittal S, Ganesh SK, Shetty NS, Gopal L. Posterior scleritis: clinical profile and imaging characteristics. Indian J Ophthalmol 1998;46:195-202.

3. Benson WE. Posterior scleritis. Surv Ophthalmol 1988;32:297316. [CrossRef]

4. Lavric A, Gonzalez-Lopez JJ, Majumder PD, Bansal N, Biswas J, Pavesio C, et al. Posterior scleritis: analysis of epidemiology, clinical factors, and risk of recurrence in a cohort of II4 patients. Ocul Immunol Inflamm 2016;24:6-I5. [CrossRef]

5. McCluskey PJ, Watson PG, Lightman S, Haybittle J, Restori M, Branley M. Posterior scleritis: clinical features, systemic associations, and outcome in a large series of patients. Ophthalmology 1999; 106:2380-6. [CrossRef]

6. Alsarhani WK, Abu El-Asrar AM. Multimodal imaging of nodular posterior scleritis: case report and review of the literature. Middle East Afr J Ophthalmol 2020;27:134-8. [CrossRef]

7. Finger PT, Perry HD, Packer S, Erdey RA, Weisman GD, Sibony $\mathrm{PA}$. Posterior scleritis as an intraocular tumour. $\mathrm{Br} \mathrm{J}$ Ophthalmol 1990;74:121-2. [CrossRef]

8. Liu AT, Luk FO, Chan CK. A case of giant nodular posterior scleritis mimicking choroidal malignancy. Indian J Ophthalmol 2015;63:919-21. [CrossRef]

9. Gonzalez-Gonzalez LA, Molina-Prat N, Doctor P, Tauber J, Sainz de la Maza M, Foster CS. Clinical features and presentation of posterior scleritis: a report of 31 cases. Ocul Immunol Inflamm 2014;22:203-7. [CrossRef]

10. Agrawal R, Lavric A, Restori M, Pavesio C, Sagoo MS. Nodular posterior scleritis: clinico-sonographic characteristics and proposed diagnostic criteria. Retina 2016;36:392-40I. [CrossRef]

I I. Sainz de la Maza M, Foster CS, Jabbur NS. Scleritis associated with systemic vasculitic diseases. Ophthalmology 1995; 102:687-92. [CrossRef]

12. Collaborative Ocular Melanoma Study Group, Boldt HC, Byrne SF, Gilson MM, Finger PT, Green RL, et al. Baseline echographic characteristics of tumors in eyes of patients enrolled in the Collaborative Ocular Melanoma Study: COMS report no. 29. Ophthalmology 2008; I 15:1390-7, 1397.el-2. [CrossRef]

13. Demirci H, Shields CL, Honavar SG, Shields JA, Bardenstein DS. Long-term follow-up of giant nodular posterior scleritis simulating choroidal melanoma. Arch Ophthalmol 2000; I I 8:1290-2.

14. Osman Saatci A, Saatci I, Kocak N, Durak I. Magnetic resonance imaging characteristics of posterior scleritis mimicking choroidal mass. Eur J Radiol 2001;39:88-91. [CrossRef]

I5. Moshari A, Cheeseman EW, McLean IW. Totally necrotic choroidal and ciliary body melanomas: associations with prognosis, 
episcleritis, and scleritis. Am J Ophthalmol 2001;131:232-6.

16. Putri CA, Salvi SM. Necrotic choroidal melanoma masquerading as scleritis. Indian J Ophthalmol 2020;68:1979-8I. [CrossRef]

17. Shields CL, Shields JA, Kiratli H, De Potter P, Cater JR. Risk factors for growth and metastasis of small choroidal melanocytic lesions. Ophthalmology 1995; 102:|351-61. [CrossRef]

18. Stephens RF, Shields JA. Diagnosis and management of cancer metastatic to the uvea: a study of 70 cases. Ophthalmology 1979;86: | 336-49. [CrossRef]

19. Augsburger JJ, Golden MI, Shields JA. Fluorescein angiography of choroidal malignant melanomas with retinal invasion. Retina
1984;4:232-41. [CrossRef]

20. Hage R, Jean-Charles A, Guyomarch J, Rahimian O, Donnio A, Merle H. Nodular posterior scleritis mimicking choroidal metastasis: a report of two cases. Clin Ophthalmol 201 1;5:87780. [CrossRef]

21. Awh C, Reichstein DA, Thomas AS. A case of giant cell arteritis presenting with nodular posterior scleritis mimicking a choroidal mass. Am J Ophthalmol Case Rep 2020; 17:100583.

22. Sielert LA, Harris AR, Pyun JM, Campbell BJ, Swan RT. Posterior scleritis. Expert Rev Ophthalmol 20 I6; I I:475-84. [CrossRef] 